\title{
Effect of Virucidals On the Presence of Severe Acute Respiratory Syndrome Coronavirus 2 (SARS-CoV-2) in Saliva
}

\author{
Richard Stanton ${ }^{1}$, Anuradha Kulasekaran ${ }^{* 2}$, Oluwajoba Adegoke ${ }^{2}$, Graca Coutinho², Jonathan Adcock ${ }^{3}$ and \\ David Thomas ${ }^{4}$
}

${ }^{1}$ Division of Infection and Immunology, School of Medicine,
Cardiff University, Cardiff, United Kingdom

${ }^{2}$ Global Medical Affairs - Respiratory. Reckitt Benckiser Healthcare Ltd.,. United Kingdom

${ }^{3} R \& D$ Manager - Respiratory and Throat. Reckitt Benckiser
Healthcare Ltd., United Kingdom.

${ }^{4}$ Advanced Therapies Group, School of Dentistry, College of Biomedical and Life Sciences, Cardiff University, Cardiff, United Kingdom.
${ }^{*}$ Corresponding author

Anuradha Kulasekaran, Global Medical Affairs - Respiratory. Reckitt Benckiser Healthcare Ltd.,. United Kingdom,

Email: anuradha.kulasekaran@rb.com

Submitted: 19 March 2021; Accepted: 08 Apr 2021;Published: 12 Apr 2021

Citation: Richard Stanton, Anuradha Kulasekaran, Oluwajoba Adegoke, Graca Coutinho, Jonathan Adcock and David Thomas(2021) Effect of Virucidals On the Presence of Severe Acute Respiratory Syndrome Coronavirus 2 (SARS-CoV-2) in Saliva. Medical \& Clinical Research 6(4): 504-505.

We read with interest the article by Belcaro G et.al. about the effect of virucidals on the presence of severe acute respiratory syndrome coronavirus 2 (SARS-CoV-2) in saliva [1]. We would like to raise major concerns on several aspects of this pilot study, including clinical methodological limitations, the use of real-time reverse transcription polymerase chain reaction (rRT-PCR) analysis of saliva, and the interpretations and conclusions drawn from the data. In our opinion, the results do not support any antiviral effects of the study products (PhytoRelief, Benzydamine, Calyptol and Baicalin), which are available as over the counter medicines or products. We would like to caution that the evidence provided is insufficient to recommend the use of these oral natural plant extracts, or other drug products, for reducing SARS-CoV-2 virus load or reducing the risks of transmission of the disease.

One of our main concerns relates to the definition and analysis of included participants. Whilst the recruited asymptomatic participants were "found to be positive" for COVID-19 at baseline, the method used for testing (e.g. quantitative PCR, lateral flow device, serology) was unspecified [1]. Furthermore, it is unclear how patients were identified as asymptomatic. Recent evidence has indicated that viral shedding of SARS-CoV-2 is reduced in a time-dependent manner following initial infection, i.e. live virus is only detected during the early phases of infection [2]. Considering the participants in this study were those with no or mild symptoms, the observed decreases in virus load may simply reflect natural decreases in viral load as the disease resolves. This would appear likely, since the study specifically excluded participants who developed symptoms on Day 3 and who, therefore, may have had sustained/increased virus titres.
We have further major concerns around how samples were collected and analysed. No information is provided about saliva collection, storage or processing, nor the RT-PCR protocol or analysis. Without these details, it is impossible to draw any conclusions as to the reliability of the data. The results are reported as the number of people with "positive" or "negative" qRT-PCR findings, though the cycle threshold $(\mathrm{Ct})$ values (or copy numbers) of samples are not provided. This is important, since the studied products may not eliminate the virus, but instead reduce the titres of virus being shed, which requires measurement of virus load rather than simply "positive" or "negative". More significantly, qRT-PCR is an inappropriate measure of antiviral efficacy in this instance because it measures virus genomes not live virus. COVID-19 genomes may represent fragments of destroyed virus, rather than shedding of live virus [3]. There is therefore no way to know whether the patients had live virus present for the products to act on in the first place. To definitively support the virucidal activity of the studied products, assays involving isolation and titration of live virus should be used.

Collectively, these limitations raise questions on the validity of the results. We believe that it is also not possible to attribute the observed effects to the study interventions, based on the methodology employed. The study products (Benzydamine solution [local non-steroidal anti-inflammatory drug], Biacalin [flavone glycoside], Calyptol vapour [eucalyptus extract], Aldiamed Certmedica lozenge [combination of glycerine, artificial sweeteners, aloe, xanthan gum and lactoferrin], and PhytoRelief lozenge [containing pomegranate, turmeric and ginger]) were administered with no information provided on the dosing regimens. 
The authors state that 3-4 times daily was "suggested"; however, no indication of compliance, nor the basis of the recommended dosing, was made, and no information was provided regarding use of concomitant therapies. Without knowing whether these instructions were followed and whether the products were used exclusively, it is not appropriate to draw definitive conclusions on the effects of the study products.

Finally, we would like to highlight that any clinical study involving humans must legally comply with international standard on ethics and consent [4]. The study does not state whether it was subject to ethical approval or that it requested consent from participants. Moreover, the authors provide no justification for sample-size, nor is any information given on blinding or randomisation methods, potentially leading to biased outcomes. Most importantly, there is no control group where participants are given a substance that will not provide an antiviral effect. Without this control, no valid conclusions regarding the antiviral efficacy of these agents can be drawn.

In the absence of robust methodological and reporting standards, the data provided do not support the antiviral activity of PhytoRelief, Calyptol and Baicalin, and conclusions that these products have antiviral effects and could be used to control the virus in vivo is misleading to both healthcare professionals and consumers. Highlighting the limitations of this study is needed: SARS-CoV-2 positive patients with mild symptoms may be recommended these products with a false belief that using these products will affect the course of their disease or reduce their infectivity.

\section{Disclosures}

David Thomas and Richard Stanton have received funding from Reckitt Benckiser Healthcare Ltd. (RB). All other authors are employees of RB.

\section{References}

1. Belcaro G, Bombardelli E, Cornelli U, Cesarone MR, Cotellese R, et al. (2020) Virucidals Control the Presence of Covid in Mouth/Saliva. Med Clin Res 5: 76-79.

2. Cevik M, Tate M, Lloyd O, Maraolo AE, Schafers J, et al. (2021) SARS-CoV-2, SARS-CoV, and MERS-CoV viral load dynamics, duration of viral shedding, and infectiousness: a systematic review and meta-analysis. Lancet Microbe 2: e13-e22.

3. Jeroen JA, David AMC, Pieter LA, Mart ML, Nisreen O, et al. (2021) Duration and key determinants of infectious virus shedding in hospitalized patients with coronavirus disease-2019 (COVID-19). Nature Communications 12: 267.

4. Sullivan PS, Sailey C, Guest JL, Guarner J, Kelley C, et al. (2020) Detection of SARS-CoV-2 RNA and Antibodies in Diverse Samples: Protocol to Validate the Sufficiency of Provider-Observed, Home-Collected Blood, Saliva, and Oropharyngeal Samples. JMIR Public Health Surveill 6: e19054.
Copyright: (C2021 Anuradha Kulasekaran, et al. This is an open-access article distributed under the terms of the Creative Commons Attribution License, which permits unrestricted use, distribution, and reproduction in any medium, provided the original author and source are credited. 